\title{
CATEGORY-ISOMORPHISMS AND ENDOMORPHISM RINGS OF MODULES
}

\author{
BY \\ KIITI MORITA
}

1. Introduction. As is well known, any two faithful modules over a simple ring are isomorphic if their endomorphism rings are isomorphic. This theorem, however, cannot be extended to the case of modules over a semi-simple ring. As a generalization of isomorphisms between modules over a ring we have the notion of semi-linear isomorphisms. But even this notion is not general enough for the above theorem to be generalized to the case of modules over a semi-simple ring $\left.{ }^{1}\right)$. It seems to the author that the most general type of condition which implies the existence of an isomorphism between the endomorphism rings of two modules is to require the existence of a categoryisomorphism carrying one of these modules into the other, and by the notion of category-isomorphisms we can obtain a generalization of the above theorem in a satisfactory form even for modules over a quasi-Frobenius ring.

Let $A$ and $B$ be associative rings each of which satisfies the minimum condition for left and right ideals and has a unit element. Let $\mathfrak{A}$ be a class of right (or left) $A$-modules and $\mathfrak{B}$ a class of right (or left) $B$-modules. A function $T$, which assigns to each module $X$ in $\mathscr{A}$ a $B$-module $T(X)$ in $\mathscr{B}$ and to each $A$-homomorphism $f: X \rightarrow X^{\prime}\left(X, X^{\prime} \in \mathfrak{A}\right)$ a $B$-homomorphism $T(f): T(X)$ $\rightarrow T\left(X^{\prime}\right)$, is called a covariant functor from the category $\mathfrak{A}$ to the category $\mathfrak{B}\left(^{2}\right)$ if the following three conditions are satisfied:

(1) If $f: X \rightarrow X$ is the identity then $T(f)$ is the identity;

$$
\begin{array}{ll}
T\left(f^{\prime} \circ f\right)=T\left(f^{\prime}\right) \circ T(f) & \text { for } f: X \rightarrow X^{\prime}, f^{\prime}: X^{\prime} \rightarrow X^{\prime \prime} ; \\
T\left(f+f^{\prime}\right)=T(f)+T\left(f^{\prime}\right) & \text { for } f, f^{\prime}: X \rightarrow X^{\prime} .
\end{array}
$$

Let $T$ and $T^{\prime}$ be two covariant functors from $\mathfrak{A}$ to $\mathfrak{B}$. A natural transformation $\Phi: T \rightarrow T^{\prime}$ is defined to be a family of $B$-homomorphisms $\Phi(X): T(X) \rightarrow T^{\prime}(X)(X \in \mathfrak{R})$ such that $T^{\prime}(f) \circ \Phi(X)=\Phi\left(X^{\prime}\right) \circ T(f)$ for each $A$-homomorphism $f: X \rightarrow X^{\prime}$. If each $\Phi(X)$ is a $B$-isomorphism of $T(X)$ onto $T^{\prime}(X)$ then $\Phi: T \rightarrow T^{\prime}$ is called a natural equivalence. If there exists a natural equivalence $\Phi: T \rightarrow T^{\prime}, T$ and $T^{\prime}$ are said to be naturally equivalent.

Received by the editors July 18,1961 .

(1) Let $A=A_{1} \oplus A_{2}$ be a semi-simple ring such that $A_{1}$ and $A_{2}$ are isomorphic respectively to the full matrix rings $(K)_{2}$ and $(K)_{3}$ over a field $K$. If we denote by $L_{i}$ the simple right ideal of $A_{i}(i=1,2)$ and put $X=L_{1} \oplus L_{2} \oplus L_{2}, Y=L_{1} \oplus L_{1} \oplus L_{2}$, then the endomorphism rings of $X$ and $Y$ are isomorphic, but there is no semi-linear isomorphism of $X$ onto $Y$.

(2) The category in which "objects" are modules in $\mathscr{A}$ and "maps" are all $A$-homomorphisms between modules in $\mathfrak{A}$ will be denoted by the same letter $\mathfrak{A}$. Here we are considering additive functors. As for functors, cf. Cartan and Eilenberg [5]. 
Let $T_{1}$ be a covariant functor from $\mathfrak{A}$ to $\mathfrak{B}$ and $T_{2}$ a covariant functor from $\mathfrak{B}$ to $\mathfrak{A}$. In case the composite functors $T_{2} T_{1}$ and $T_{1} T_{2}$ are naturally equivalent to the identity functor, $T_{1}$ (resp. $T_{2}$ ) is called a category-isomorphism from $\mathfrak{A}$ onto $\mathfrak{B}$ (resp. from $\mathfrak{B}$ onto $\mathfrak{A})\left({ }^{3}\right)$.

For a ring $C$ we shall denote always by $\mathfrak{M}_{C}$ the category of all right $C$ modules and by $\operatorname{End}_{C}(M)$ the $C$-endomorphism ring of a right $C$-module $M$.

With these notations we have

Proposition 1.1. Let $X$ be a right $A$-module and $Y$ a right $B$-module. If there exists a category-isomorphism $T$ from $\mathfrak{M}_{A}$ onto $\mathfrak{M}_{B}$ such that $T(X)$ is $B$-isomorphic to $Y$, then $\operatorname{End}_{A}(X)$ is ring-isomorphic to $\operatorname{End}_{B}(Y)$.

Indeed, the correspondence $f \rightarrow \psi \circ T(f) \circ \psi^{-1}$ for $f \in \operatorname{End}_{A}(X)$ gives a ring-isomorphism of $\operatorname{End}_{A}(X)$ onto $\operatorname{End}_{B}(Y)$ where $\psi$ is a $B$-isomorphism of $T(X)$ onto $Y$.

The present paper is concerned with the problem: For what kind of rings $A$ and $B$ does the converse of Proposition 1.1 hold?

In discussing this problem we shall first restrict ourselves to the case of faithful modules. It can be shown that if $A$ and $B$ are quasi-Frobenius rings and if a right $A$-module $X$ and a right $B$-module $Y$ are faithful modules which are direct sums of finitely generated submodules then the converse of Proposition 1.1 holds. However, we can establish a more general and more precise result.

We shall say that a subring $C$ of $\operatorname{End}_{A}(X)$ is strongly dense in $\operatorname{End}_{\Lambda}(X)$ if the following two conditions are satisfied:

(1) $C$ is dense in $\operatorname{End}_{A}(X)$ in the ordinary sense; that is, for any finite number of elements $x_{1}, \cdots, x_{m}$ in $X$ and for any endomorphism $f \in \operatorname{End}_{\Delta}(X)$ there exists $g \in C$ such that $g\left(x_{i}\right)=f\left(x_{i}\right)$ for $i=1,2, \cdots, m$.

(2) For each finitely generated $A$-submodule $M$ of $X$ which is a direct summand of $X$ there exists $g \in C$ such that $g$ is a projection of $X$ onto $M$.

Here we shall recall some definitions. Let $A$ be a ring which has a unit element and satisfies the minimum condition for left and right ideals. Then $A$ is called quasi-Frobenius if $\operatorname{Hom}_{A}\left(\operatorname{Hom}_{A}(X, A), A\right) \cong X$ holds for every finitely generated left or right $A$-module $X$ where $\operatorname{Hom}_{A}(Y, A)$ for a left (resp. right) $A$-module $Y$ means the right (resp. left) $A$-module which consists of all $A$-homomorphisms of $Y$ into $A$ with the usual compositions: $(\alpha+\beta)(y)=\alpha(y)+\beta(y), \quad(\alpha a)(y)=\alpha(y) a$ (resp. $(a \alpha)(y)=a(\alpha(y)))$, for $\alpha, \beta$ $\in \operatorname{Hom}_{A}(Y, A), a \in A, y \in Y . A$ is called generalized uni-serial if every left ideal $A e$ as well as every right ideal $e A$ generated by a primitive idempotent element $e$ possesses only one composition series. $A$ is called uni-serial if $A$ is generalized uni-serial and if $A$ is a direct sum of two-sided ideals each of which is a primary ring. Semi-simple rings are uni-serial, and uni-serial rings

( $\left.{ }^{3}\right)$ As for category-isomorphisms cf. Morita [8], in which they are introduced under the term of isomorphisms. 
are quasi-Frobenius, and group rings of finite groups over a field are also quasi-Frobenius.

Now we can state our main theorem.

Theorem 1.2. Let $A$ and $B$ be quasi-Frobenius rings. Let $X$ and $Y$ be respectively a faithful right $A$-module and a faithful right $B$-module such that $X$ and $Y$ are direct sums of finitely generated submodules. Let $C$ be a strongly dense subring of $\operatorname{End}_{A}(X)$ and $D$ a strongly dense subring of $\operatorname{End}_{B}(Y)$. If $\Phi$ is a ringisomorphism of $C$ onto $D$, then there exists a category-isomorphism $T$ from $\mathfrak{M}_{A}$ onto $\mathfrak{M}_{B}$ such that $T(X)$ is $B$-isomorphic to $Y$ and

$$
\Phi(f)=\psi \circ T(f) \circ \psi^{-1}
$$$$
\text { for } f \in C
$$

holds with a B-isomorphism $\psi$ of $T(X)$ onto $Y$.

As a special case where $C=\operatorname{End}_{A}(X)$ and $D=\operatorname{End}_{B}(Y)$ Theorem 1.2 contains a solution to the problem proposed above in its precise form.

In applications of Theorem 1.2 it will be useful to note that any module over a generalized uni-serial ring is a direct sum of cyclic submodules (cf. Nakayama [11]) and hence a direct sum of finitely generated submodules.

In case $A$ and $B$ are self-basic, that is, each of them coincides with its basic ring, Theorem 1.2 can be brought into another form:

TheOREM 1.3. Let $A$ and $B$ be self-basic quasi-Frobenius rings. Let $X, Y$, $C, D$ be as in Theorem 1.2. Suppose that $\Phi$ is a ring-isomorphism of $C$ onto $D$. Then there exists a semi-linear isomorphism $\omega$ of $X$ onto $Y$ with a ring-isomorphism $\theta$ of $A$ onto $B\left({ }^{4}\right)$ such that

$$
\Phi(f)=\omega \circ f \circ \omega^{-1}, \quad \text { for } f \in C .
$$

As is well known, any dense subring of the ring of all linear transformations of a vector space over a division ring is strongly dense in our sense if it contains a nonzero linear transformation of finite rank. Accordingly it is seen that the isomorphism theorem for primitive rings with minimal left ideals (cf. Jacobson [7]) may be obtained as a special case of Theorem 1.3; we have only to notice that a division ring is self-basic, generalized uni-serial and quasiFrobenius. Likewise Shoda's theorem [14], Asano's theorem [1], and Baer's theorem [4], which are concerned with endomorphism rings of modules over a commutative or completely primary uni-serial ring, are also special cases of our Theorem 1.3.

In the above theorems we have dealt with the case of faithful modules. In case we admit $X$ and $Y$ to be nonfaithful modules, we must restrict ourselves to the case where $A$ and $B$ are similar $\left(^{5}\right)$. This restriction is justified by the

(4) That is, $\omega$ is a one-to-one mapping of $X$ onto $Y$ such that $\omega\left(x+x^{\prime}\right)=\omega(x)+\omega\left(x^{\prime}\right)$, $\omega(x a)=\omega(x) \theta(a)$ for $x, x^{\prime} \in X, a \in A$.

(5) If the basic rings of $A$ and $B$ are isomorphic, we shall say that $A$ and $B$ are similar. 
following two facts: (1) if there exists a category-isomorphism from $\mathfrak{M}_{A}$ onto $\mathfrak{M}_{B}$ then $A$ and $B$ are similar (cf. Morita [8, Theorem 7.5]), and (2) for a nonzero two-sided ideal $I$ of $A$ the residue class ring $A / I$ is not similar to $A$ but $\operatorname{End}_{A}(X) \cong \operatorname{End}_{A / I}(X)$ for any right $A / I$-module $X$. Our results in this case will be stated in $\S 7$.

The types of rings to which $A$ and $B$ are restricted in the above theorems are shown (under some condition) to be general enough for the conclusions in these theorems to hold. From this point of view we have a new characterization of quasi-Frobenius rings $(\$ 6)$.

If in Theorems 1.2 and 1.3 we impose on $X$ and $Y$ a further restriction that $X$ and $Y$ should be fully faithful in the sense defined in $\S 3$ then these theorems remain true for any rings $A$ and $B$ with a slight condition which is satisfied for any algebras over a commutative field; these results are stated in $\S 3$ as Theorems 3.2 and 3.3 from which Theorems 1.2 and 1.3 will be deduced as immediate corollaries.

Throughout this paper the meanings of the notations used in this introduction will be retained; thus, $A$ and $B$ are always assumed to be associative rings each of which has a unit element and satisfies the minimum condition for left and right ideals. Modules over a ring are assumed to be unital in the sense that the unit element of the ring acts on the modules as the identity operator.

2. Category-isomorphisms. Let us denote by 1 the unit element of $A$. Then there exist mutually orthogonal primitive idempotent elements $e_{k, i}$, $k=1, \cdots, m ; i=1, \cdots, r(k)$ such that

$$
1=\sum_{k=1}^{m} \sum_{i=1}^{r(k)} e_{k, i} ; \quad A e_{k, i} \cong A e_{l, j} \quad \text { if and only if } k=e .
$$

Let us put

$$
A^{0}=e A e, \quad e=\sum_{k=1}^{m} e_{k, 1} .
$$

$A^{0}$ is called the basic ring of $A$; the basic ring of $A$ is determined by $A$ uniquely up to an inner automorphism (cf. Osima [13]).

Let us set

$$
\begin{array}{ll}
P_{1}(X)=X \otimes_{A} A e, & \text { for } X \in \mathfrak{M}_{A}, \\
P_{2}\left(X^{0}\right)=\operatorname{Hom}_{A}{ }^{0}\left(A e, X^{0}\right), & \text { for } X^{0} \in \mathfrak{M}_{A}{ }^{0},
\end{array}
$$

where $A e$ is considered as a two-sided $A-A^{0}$-module. Then, as is shown in Morita $[8, \S 7], P_{1}$ is a category-isomorphism from $\mathfrak{M}_{A}$ onto $\mathfrak{M}_{A^{0}}$ and $P_{2}$ a category-isomorphism from $\mathfrak{M}_{A^{0}}$ onto $\mathfrak{M}_{A}$, and the composite functors $P_{2} P_{1}$ and $P_{1} P_{2}$ are naturally equivalent to the identity functor.

For $B$ we shall construct the corresponding functors: 


$$
\begin{aligned}
& Q_{1}(Y)=Y \otimes_{B} B e^{\prime} \\
& Q_{2}\left(Y^{0}\right)=\operatorname{Hom}_{B^{0}}\left(B e^{\prime}, Y^{0}\right),
\end{aligned}
$$

for $Y \in \mathfrak{M}_{B}$, for $Y^{0} \in \mathfrak{M}_{B^{0}}$

where $e^{\prime}$ is defined similarly as $e$ for $A, B=e^{\prime} B e^{\prime}$ is the basic ring of $B$, and $B e^{\prime}$ is considered as a two-sided $B-B^{0}$-module. The composite functors $Q_{2} Q_{1}$ and $Q_{1} Q_{2}$ are naturally equivalent to the identity functor, and $Q_{1}: \mathfrak{M}_{B} \rightarrow \mathfrak{M}_{B^{0}}$,

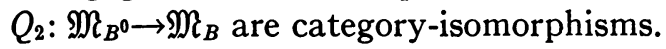

Let $T$ be any category-isomorphism from $\mathfrak{M}_{A}$ onto $\mathfrak{M}_{B}$. Then

$$
T^{0}=Q_{1} T P_{2}: \mathfrak{M}_{A^{0}} \rightarrow \mathfrak{M}_{B^{0}}
$$

is a category-isomorphism and $T$ is naturally equivalent to a categoryisomorphism $Q_{2} T^{0} P_{1}$. Thus the diagram

$$
\begin{array}{cc}
\mathfrak{M}_{A} \stackrel{T}{\longrightarrow} \mathfrak{M}_{B} \\
P_{2} \uparrow \downarrow P_{1} \quad Q_{1} \downarrow Q_{2} \\
\mathfrak{M}_{A^{0}} \longrightarrow \mathfrak{M}_{B^{0}}
\end{array}
$$

is commutative in the sense that the composite functors indicated by arrows are naturally equivalent if they are defined over the same category and have values in the other same category.

Thus the study of category-isomorphisms from $\mathfrak{M}_{A}$ onto $\mathfrak{M}_{B}$ is reduced to that of category-isomorphisms from $\mathfrak{M}_{A^{0}}$ onto $\mathfrak{M}_{B^{0}}$. As for the latter the following theorem is fundamental.

THEOREM 2.1. Let $\mathfrak{a}$ be a class of right A-modules containing $A$ as a right $A$-module and $B$ a class of right $B$-modules containing $B$ as a right $B$-module. Let $T_{1}$ and $T_{2}$ be category-isomorphisms from $\mathfrak{A}$ onto $\mathfrak{B}$ and from $\mathfrak{B}$ onto $\mathfrak{A}$ such that $T_{2} T_{1}$ and $T_{1} T_{2}$ are naturally equivalent to the identity functor. Suppose that there is an $A$-isomorphism $\sigma: A \rightarrow T_{2}(B)$. Then there exists a ring-isomorphism $\theta$ from $A$ onto $B$ such that

$$
T_{2}\left(\psi_{\theta(a)}\right)=\sigma \circ \phi_{a} \circ \sigma^{-1},
$$$$
\text { for } a \in A
$$

holds. (1) is equivalent to

$$
\sigma(a u)=\theta(a) \sigma(u), \quad \text { for } u \in A, a \in A .
$$

Here $\phi_{a}: A \rightarrow A$ is a right $A$-homomorphism defined by $\phi_{a}(x)=a x$ for $x \in A$ and $\psi_{b}: B \rightarrow B$ a right $B$-homomorphism defined by $\psi_{b}(y)=b y$ for $y \in B$, and bv is defined to be $T_{2}\left(\psi_{b}\right) v$ for $v \in T_{2}(B), b \in B$.

In this case $V=T_{2}(B)$ becomes a two-sided $B-A$-module and $T_{1}$ is naturally equivalent to the functor $\operatorname{Hom}_{A}(V$,$) . Furthermore$

$$
\Gamma(X): X \rightarrow \operatorname{Hom}_{A}(V, X)
$$

for $X \in \mathfrak{A}$

defined by $\Gamma(X)(x)=\phi_{x} \circ \sigma^{-1}$ is a semi-linear isomorphism with a ring-isomor- 
phism $\theta$ of $A$ onto $B$, that is, $\Gamma(X)(x a)=[\Gamma(X)(x)] \theta(a)$, where $\phi_{x}: A \rightarrow X$ is defined by $\phi_{x}(a)=x a$ for $x \in X, a \in A$.

Proof. Since $\sigma^{-1} \circ T_{2}\left(\psi_{b}\right) \circ \sigma$ is an $A$-endomorphism of the right $A$-module $A$, there exists uniquely an element of $A$, which will be denoted by $\chi(b)$, such that $\sigma^{-1} \circ T_{2}\left(\psi_{b}\right) \circ \sigma=\phi_{\chi(b)}$. Then $\chi$ is easily shown to be a ring-isomorphism of $B$ onto $A$. If we put $\theta=\chi^{-1}$, we have the first part of the theorem.

The second part is proved in Morita [8, Theorem 3.1]. As for the last part we have

$$
\begin{aligned}
{[\Gamma(X)(x a)](v) } & =\left(\phi_{x a} \circ \sigma^{-1}\right)(v)=(x a)\left[\sigma^{-1}(v)\right] \\
& =x\left[a\left(\sigma^{-1}(v)\right)\right]=x\left[\sigma^{-1}(\theta(a) v)\right]=\left(\phi_{x} \circ \sigma^{-1}\right)(\theta(a) v) \\
& =[\Gamma(X)(x)](\theta(a) v)=[(\Gamma(X)(x)) \theta(a)](v) .
\end{aligned}
$$

This completes the proof.

In Theorem 2.1 , if we put $v_{0}=\sigma(1)$, then we have

$$
\begin{aligned}
V & =\left\{v_{0} a \mid a \in A\right\}, \quad v_{0} a=0 \text { if and only if } a=0, & \\
v_{0} a & =\theta(a) v_{0} & \text { for } a \in A .
\end{aligned}
$$

Conversely, for a given ring-isomorphism $\theta$ of $A$ onto $B$ we can construct a two-sided $B-A$-module $V$ by the formulas (7) and (8). Then $\sigma: A \rightarrow V$ defined by $\sigma(a)=v_{0} a$ is an $A$-isomorphism satisfying (5), and the covariant functor $T$ defined by

$$
\begin{gathered}
T(X)=\operatorname{Hom}_{A}(V, X), \quad \text { for } X \in \mathfrak{M}_{\Lambda}, \\
{[T(f)](\alpha)=f \circ \alpha, \quad \text { for } \alpha \in \operatorname{Hom}_{A}(V, X), f \in \operatorname{Hom}_{A}\left(X, X^{\prime}\right)}
\end{gathered}
$$

is a category-isomorphism from $\mathfrak{M}_{A}$ onto $\mathfrak{M}_{B}$ by Morita [8, Theorem 3.4] and the ring-isomorphism associated with $T$ by Theorem 2.1 is equal to $\theta$.

LеммA 2.2. With respect to the category-isomorphism $T$ defined above we have

$$
T(f)=\Gamma(X) \circ f \circ \Gamma(X)^{-1}, \quad \text { for } f \in \operatorname{End}_{\Lambda}(X),
$$

where $X \in \mathfrak{M}_{\Delta}$ and $\Gamma(X)$ is defined by (6).

Proof. For the sake of brevity we set $\omega_{1}=\Gamma(X)$ and $S(f)=\Gamma(X) \circ f \circ \Gamma(X)^{-1}$. Let $\alpha \in \operatorname{Hom}_{\Delta}(V, X), a \in A$, and put $x=\alpha\left(v_{0}\right)$. Then we have

$$
\begin{aligned}
{\left[\omega_{1}(x)\right]\left(v_{0} a\right) } & =\left(\phi_{x} \circ \sigma^{-1}\right)\left(v_{0} a\right)=\phi_{x}\left(\sigma^{-1}\left(v_{0} a\right)\right)=\phi_{x}(a)=x a, \\
\alpha\left(v_{0} a\right) & =\left[\alpha\left(v_{0}\right)\right] a=x a,
\end{aligned}
$$

and hence $\omega_{1}(x)=\alpha$. Therefore

$$
S(f)(\alpha)=\left(\omega_{1} \circ f \circ \omega_{1}^{-1}\right)(\alpha)=\omega_{1}(f(x))
$$

and consequently 


$$
[S(f)(\alpha)]\left(v_{0} a\right)=\left[\omega_{1}(f(x))\right]\left(v_{0} a\right)=f(x) a .
$$

On the other hand, we have

$$
[T(f)(\alpha)]\left(v_{0} a\right)=(f \circ \alpha)\left(v_{0} a\right)=f\left(\alpha\left(v_{0} a\right)\right)=f(x a)=f(x) a .
$$

This completes our proof.

LEMMA 2.3. Let $\omega$ be a semi-linear isomorphism of a right $A$-module $X$ onto $a$ right $B$-module $Y$ with a ring-isomorphism $\theta$ of $A$ onto $B$. If we put $\psi$ $=\omega \circ \Gamma(X)^{-1}$, then $\psi$ is a $B$-isomorphism of $T(X)$ onto $Y$ and

$$
\omega \circ f \circ \omega^{-1}=\psi \circ T(f) \circ \psi^{-1} \quad \text { for } f \in \operatorname{End}_{A}(X) \text {. }
$$

Proof. This is an immediate consequence of Lemma 2.2.

Now let $A$ and $B$ be self-basic rings. Then for any category-isomorphism $T_{2}$ from $\mathfrak{M}_{B}$ onto $\mathfrak{M}_{A}$ there exists always an $A$-isomorphism $\sigma: A \rightarrow T_{2}(B)$; this is seen from the fact that the projectivity and indecomposability of modules are preserved under any category-isomorphism (cf. Morita $[8, \S 13]$ ). Therefore any category-isomorphism from $\mathfrak{M}_{A}$ onto $\mathfrak{M}_{B}$ is determined by a ring-isomorphism $\theta$ of $A$ on to $B$.

3. Main theorem. In this section we shall state our main theorem in a more general setting.

We shall say that a right $A$-module $X$ is fully faithful, if every indecomposable projective right $A$-module as well as every indecomposable injective right $A$-module is $A$-isomorphic to a direct summand of $X$. Then we have clearly (e.g., cf. [8, Theorems 14.1 and 16.5]).

Proposition 3.1. A is a quasi-Frobenius ring if and only if every faithful right $A$-module is fully faithful.

We are now in a position to state our main theorem in a more general form.

Theorem 3.2. Assume that every indecomposable injective right A-module and every indecomposable injective right $B$-module are finitely generated. Let $X$ be a fully faithful right $A$-module and $Y$ a fully faithful right $B$-module, and let $X$ and $Y$ be direct sums of finitely generated submodules. Let $C$ be a strongly dense subring of $\operatorname{End}_{A}(X)$ and $D$ a strongly dense subring of $\operatorname{End}_{B}(Y)$. If $\Phi$ is a ring-isomorphism of $C$ onto $D$, then there exists a category-isomorphism $T$ from $\mathfrak{M}_{A}$ onto $\mathfrak{M}_{B}$ such that $T(X)$ is $B$-isomorphic to $Y$ and

$$
\Phi(f)=\psi \circ T(f) \circ \psi^{-1}
$$

for $f \in C$

holds with a B-isomorphism $\psi$ of $T(X)$ onto $Y$.

The following theorem corresponds to Theorem 1.3.

Theorem 3.3. Let $A, B, X, Y, C$, and $D$ be as in Theorem 3.2. Assume further that $A$ and $B$ are self-basic. If $\Phi$ is a ring-isomorphism of $C$ onto $D$, then 
there exists a semi-linear isomorphism $\omega$ of $X$ onto $Y$ with a ring-isomorphism $\theta$ of $A$ onto $B$ such that

$$
\Phi(f)=\omega \circ f \circ \omega^{-1}, \quad \text { for } f \in C .
$$

From Proposition 3.1 it follows that Theorems 1.2 and 1.3 are special cases of Theorems 3.2 and 3.3 respectively. We shall prove here

Proposition 3.4. Theorem 3.2 can be deduced from Theorem 3.3.

Let $A, B, X, Y, C$ and $D$ be the same as in Theorem 3.2, and let $\Phi$ be a ring-isomorphism of $C$ onto $D$. With the notations used at the beginning of $\$ 2$ we set

$$
\begin{array}{ll}
X^{0}=P_{1}(X), \quad Y^{0}=Q_{1}(Y), & \\
C^{0}=\left\{P_{1}(f) \mid f \in C\right\}, \quad D^{0}=\left\{Q_{1}(g) \mid g \in D\right\}, & \text { for } f \in C .
\end{array}
$$

Then $X^{0}$ and $Y^{0}$ are fully faithful modules over $A^{0}$ and $B^{0}$ respectively, and they are direct sums of finitely generated submodules. $C^{0}$ and $D^{0}$ are strongly dense subrings of $\operatorname{End}_{A^{0}}\left(X^{0}\right)$ and $\operatorname{End}_{B^{0}}\left(Y^{0}\right)$ respectively. $\Phi_{0}$ is a ring-isomorphism of $C^{0}$ onto $D^{0}$. Moreover, $A^{0}$ and $B^{0}$ are self-basic rings such that every indecomposable injective right module over $A^{0}$ (resp. $B^{0}$ ) is finitely generated.

Let us now assume that Theorem 3.3 is true. Then there exists a semilinear isomorphism $\omega_{0}$ of $X^{0}$ onto $Y^{0}$ such that $\Phi_{0}\left(P_{1}(f)\right)=\omega_{0} \circ P_{1}(f) \circ \omega_{0}^{-1}$ for $f \in C$. Hence by Lemma 2.3 there exists a category-isomorphism $T_{0}$ from $\mathfrak{M}_{A^{0}}$ onto $\mathfrak{M}_{B^{0}}$ such that

$$
\Phi_{0}\left(P_{1}(f)\right)=\psi_{0} \circ T_{0}\left(P_{1}(f)\right) \circ \psi_{0}^{-1}
$$

for $f \in C$

holds with a $B^{0}$-isomorphism $\psi_{0}: T_{0}\left(X^{0}\right) \cong Y^{0}$.

From (9) and (10) we get

$$
\left(Q_{2} Q_{1}\right)(\Phi(f))=Q_{2}\left(\psi_{0}\right) \circ\left(Q_{2} T_{0} P_{1}\right)(f) \circ Q_{2}\left(\psi_{0}\right)^{-1} .
$$

Now let $\mu$ be the natural equivalence from the identity functor to $Q_{2} Q_{1}$. Then we have

$$
\left(Q_{2} Q_{1}\right)(\Phi(f))=\mu(Y) \circ \Phi(f) \circ \mu(Y)^{-1}
$$

Hence, if we put

$$
T=Q_{2} T_{0} P_{1}, \quad \psi=\mu(Y)^{-1} \circ Q_{2}\left(\psi_{0}\right),
$$

then $T$ is a category-isomorphism from $\mathfrak{M}_{A}$ onto $\mathfrak{M}_{B}, \psi$ is a $B$-isomorphism of $T(X)$ onto $Y$, and 


$$
\Phi(f)=\psi \circ T(f) \circ \psi^{-1}
$$

for $f \in C$.

Thus we have proved that Theorem 3.3 implies Theorem 3.2.

\section{Some lemmas.}

Lemma 4.1. Let $X$ be a faithful right $A$-module which is decomposed into a direct sum of two submodules $X_{0}$ and $X_{1}$ such that $X_{0}$ is $A$-isomorphic to $A$ as a right $A$-module. Let $C$ be any dense subring of $\operatorname{End}_{A}(X)$. Suppose that $C$ contains $a$ projection $e_{0}$ from $X$ onto $X_{0}$. Then the left $C$-module $X$ is $C$-isomorphic to $C e_{0}$, and any $C$-endomorphism of $X$ is obtained by the right multiplication of an element of $A$.

Proof. We assume that $X_{1}=\left(1-e_{0}\right) X$. Let $x_{0}$ be the element of $X_{0}$ corresponding to the unit element of $A$ by the given isomorphism of $X_{0}$ onto $A$. We put $\alpha\left(x_{0} a\right)=a$ for $a \in A ; \alpha(x)=0$ for $x \in X_{1}$. Then we have $\alpha \in \operatorname{Hom}_{A}(X, A)$. Then for $x^{\prime} \in X$ the correspondence $x \rightarrow x^{\prime} \alpha(x)$ defines an $A$-endomorphism of $X$ which will be denoted by $\sigma\left(x^{\prime}\right)$; we have

$$
x^{\prime}[\alpha(x)]=\sigma\left(x^{\prime}\right) x, \quad \sigma\left(x^{\prime}\right) \in \operatorname{End}_{A}(X) .
$$

For $\sigma$ we have $\sigma\left(x+x^{\prime}\right)=\sigma(x)+\sigma\left(x^{\prime}\right), \sigma(c x)=c \sigma(x)$ for $c \in \operatorname{End}_{A}(X)$. Moreover, we have $\sigma\left(x_{0}\right)=e_{0}, \sigma(x) e_{0}=\sigma(x)$ for $x \in X$, since

$$
\sigma\left(x_{0}\right)\left(x_{0} a\right)=x_{0} \alpha\left(x_{0} a\right)=x_{0} a
$$

for $a \in A$,

and

$$
\sigma\left(x_{0}\right)(x)=x_{0} \alpha(x)=0 \quad \text { for } x \in X_{1} ;
$$

(2) $\sigma(x) x^{\prime}=x\left[\alpha\left(x^{\prime}\right)\right]=x\left(\alpha\left(e_{0} x^{\prime}\right)\right)=\sigma(x)\left[e_{0} x^{\prime}\right]=\left(\sigma(x) e_{0}\right)\left(x^{\prime}\right)$ for any $x^{\prime} \in X$.

Thus $\sigma$ is an $\operatorname{End}_{A}(X)$-homomorphism of $X$ into $\operatorname{End}_{A}(X) e_{0}$. Furthermore, $\sigma$ is an $\operatorname{End}_{A}(X)$-isomorphism; (1) for $x \neq x^{\prime}$ we have $\sigma(x) \neq \sigma\left(x^{\prime}\right)$ because $x=x \alpha\left(x_{0}\right)=\sigma(x) x_{0} ;(2)$ for any $f \in \operatorname{End}_{A}(X)$ we have $\sigma\left(f x_{0}\right)=f \sigma\left(x_{0}\right)=f e_{0}$ and hence $\sigma$ is onto.

Now let $f \in \operatorname{End}_{A}(X)$. Then, since $C$ is dense in $\operatorname{End}_{A}(X)$, there exists $g \in C$ such that $g\left(x_{0}\right)=f\left(x_{0}\right)$. For this $g$ we have $g e_{0}=g \sigma\left(x_{0}\right)=\sigma\left(g x_{0}\right)=\sigma\left(f x_{0}\right)$ $=f \sigma\left(x_{0}\right)=f e_{0}$. Hence $C e_{0}=\left[\operatorname{End}_{A}(X)\right] e_{0}$. This proves the first part.

To prove the second part, let $\lambda$ be any $C$-endomorphism of $X$. If we put $a=\alpha\left(\lambda\left(x_{0}\right)\right)$, then for any $x \in X$ we have $x a=x\left(\alpha\left(\lambda\left(x_{0}\right)\right)\right)=\sigma(x) \lambda\left(x_{0}\right)=\lambda\left(\sigma(x) x_{0}\right)$ $=\lambda\left(x \alpha\left(x_{0}\right)\right)=\lambda(x)$. (Cf. Morita $\left.[8, \S 3]\right)$.

Lemma 4.2. Let $X$ be a right $A$-module which is decomposed into a direct sum of finitely generated $A$-submodules. Let $C$ be a strongly dense subring of $\operatorname{End}_{A}(X)$. If $e$ is a primitive idempotent element of $C$, then $e$ is primitive also in End $_{A}(X)$ and $e X$ is a finitely generated, indecomposable $A$-module.

Proof. From the assumption it follows that $X$ is a direct sum of indecomposable, finitely generated $A$-submodules $X_{\lambda}(\lambda \in \Lambda): X=\Sigma \oplus X_{\lambda}$. Then for each $\lambda$ there exists an idempotent element $e_{\lambda} \in C$ such that $X_{\lambda}=e_{\lambda} X$. By 
Azumaya [2, Theorem 1] there exist some $e_{\lambda}$ and an idempotent element $f \in C$ such that $e$ is one-to-one on $e_{\lambda} X$, and $e e_{\lambda} X=f X$ and $f$ is primitive in $\operatorname{End}_{\mathbf{A}}(X)$. Hence we have

$$
e f=f, \quad f e e_{\lambda}=e e_{\lambda} .
$$

Furthermore, we have

$$
\begin{gathered}
e=e f e+(e-e f e) \\
(e f e)(e f e)=e f e, \quad \text { efe }(e-e f e)=(e-e f e) e f e=0 .
\end{gathered}
$$

Since $e \in C, f \in C$ and $e$ is primitive in $C$, we have $e=e f e$; otherwise we would have $e f e=0$ and hence $e e_{\lambda}=(e f e) e_{\lambda}=0$ which contradicts $e e_{\lambda} X=f X \neq 0$. Hence $f e=(e f) e=e$. Thus we get $e f=f, f e=e$. This shows that $e X=f X$. Therefore $e X$ is a finitely generated, indecomposable $A$-submodule and $e$ is primitive in $\operatorname{End}_{A}(X)$.

Lemma 4.3. Let $X$ and $C$ be as in Lemma 4.2. Let e be an idempotent element of $C$ such that $e X$ is finitely generated. Then $e$ is a sum of a finite number of mutually orthogonal primitive idempotent elements of $C$.

Proof. Let $e X=X_{1} \oplus \cdots \oplus X_{\text {s }}$ be a decomposition of $e X$ into a direct sum of indecomposable, finitely generated submodules. We shall denote by $e_{i}$ the $A$-endomorphism of $X$ which is identity on $X_{i}$ and zero on $(1-e) X$ $\oplus \sum_{j \neq i} \oplus X_{j}$. Then we have $e=e_{1}+\cdots+e_{s}$. Since $e X$ is finitely generated and $C$ is dense in $\operatorname{End}_{A}(X)$, there exist $h_{i} \in C$ such that $h_{i} x=e_{i} x$ for all $x \in e X$. Then we have $e_{i} x=h_{i} e x$ for each $x \in X$ and hence $e_{i}=h_{i} e$. Since $e, h_{i} \in C$, we have $e_{i} \in C$.

5. Proof of the main theorem. As was shown in $\S 3$, we have only to prove Theorem 3.3.

For this purpose, let $A$ and $B$ be self-basic rings such that every indecomposable injective right $A$-module (resp. $B$-module) is finitely generated. Let $X$ (resp. $Y$ ) be a fully faithful right $A$-module (resp. $B$-module) which is a direct sum of finitely generated submodules. Let $C$ be a strongly dense subring of $\operatorname{End}_{A}(X)$ and $D$ a strongly dense subring of $\operatorname{End}_{B}(Y)$. Furthermore, let $\Phi$ be a ring-isomorphism of $C$ onto $D$.

We shall first deal with the case where $X$ and $Y$ are finitely generated.

\section{Lemma 5.1. Theorem 3.3 holds if $X$ and $Y$ are finitely generated.}

Proof. Since $C$ and $D$ are dense in $\operatorname{End}_{A}(X)$ and $\operatorname{End}_{B}(Y)$ respectively and $X$ and $Y$ are finitely generated, we have $C=\operatorname{End}_{A}(X)$ and $D=\operatorname{End}_{B}(Y)$. Since $X$ and $Y$ are fully faithful and finitely generated, by Morita [8, Theorems 16.3 and 16.4] the left $C$-module $X$ as well as the left $D$-module $Y$ is finitely generated, faithful, projective and injective $\left({ }^{(}\right)$. tive.

$\left.{ }^{(}\right)$In case $X$ is not finitely generated, the left $C$-module $X$ is still projective but not injec- 
Let us set

$$
c * y=\Phi(c) y \quad \text { for } y \in Y, c \in C .
$$

Then $Y$ is a left $C$-module which is finitely generated, faithful, projective and injective. Since the $C$-endomorphism ring of $X$ (resp. the $D$-endomorphism ring of $Y$ ) is inverse-isomorphic to $A$ (resp. $B$ ) and $A$ (resp. $B$ ) is self-basic, the left $C$-modules $X$ and $Y$ are $C$-isomorphic to $C e_{1}$ and $C e_{2}$ where each of $e_{1}$ and $e_{2}$ is a sum of a finite number of mutually orthogonal primitive idempotent elements of $C$ such that any two of them generate nonisomorphic left ideals of $C$. Here it is to be noted that the ring $C$ is semi-primary in the sense that its radical is nilpotent and the residue class ring modulo its radical satisfies the minimum condition for left and right ideals. Hence the semi-simple part (= socle) of an indecomposable injective left $C$-module is simple and two injective left $C$-modules are $C$-isomorphic if and only if their semi-simple parts are $C$-isomorphic $\left({ }^{7}\right)$. In the present situation, each of the semi-simple parts of $C e_{1}$ and $C e_{2}$ is a direct sum of a finite number of mutually nonisomorphic simple left $C$-modules which exhaust all types of simple left $C$-modules contained in $C$. Therefore the semi-simple parts of $C e_{1}$ and $C e_{2}$ are $C$-isomorphic, and consequently $C e_{1}$ and $C e_{2}$ are $C$-isomorphic $\left({ }^{8}\right)$. Let $\omega$ be a $C$-isomorphism of $X$ onto $Y$. Then we have

$$
\omega(c x)=c * \omega(x)=\Phi(c) \omega(x) \quad \text { for } x \in X, c \in C .
$$

On the other hand, for an element $a$ of $A$ the mapping $y \rightarrow \omega\left(\omega^{-1}(y) a\right)$ defines a $D$-endomorphism of $Y$ and hence there exists an element $\theta(a)$ of $B$ such that $\omega\left(\omega^{-1}(y) a\right)=y \theta(a)$. Thus we have $\omega(x a)=\omega(x) \theta(a)$. It is easy to see that $\theta$ is a ring-isomorphism of $A$ onto $B$. This completes the proof of Lemma 5.1.

We shall now return to the general case where $X$ and $Y$ are not necessarily finitely generated.

Let $e$ and $e^{\prime}$ be idempotent elements of $C$ such that $e X$ is $A$-isomorphic to the right $A$-module $A$ and $e^{\prime} X$ is a direct sum of a finite number of mutually nonisomorphic indecomposable injective right $A$-modules which exhaust all types of indecomposable injective right $A$-modules; the existence of $e$ and $e^{\prime}$ is assured by the assumption that $C$ is strongly dense and $X$ is fully faithful. Let $f$ and $f^{\prime}$ be idempotent elements of $D$ having the properties corresponding to $e$ and $e^{\prime}$ respectively.

Then by Lemma 4.3 each of $f$ and $f^{\prime}$ is a direct sum of a finite number of mutually orthogonal primitive idempotent elements of $D$, and hence each of

( ${ }^{7}$ Theorem 6.1 in Morita [8] holds for semi-primary rings in the above sense (with the exception of $3^{\circ}$ ).

(8) From the fact that $C e_{i}$ is a direct sum of a finite number of mutually nonisomorphic indecomposable injective left ideals of $C$, it can be shown that $C e_{i}$ is a minimal faithful left $C$ module in the sense that any faithful left $C$-module has a direct summand which is $C$-isomorphic to $C e_{i}(i=1,2)$. From this we have another proof for the proposition that $C e_{1}$ and $C e_{2}$ are $C$ isomorphic. 
$\Phi^{-1}(f)$ and $\Phi^{-1}\left(f^{\prime}\right)$ is a direct sum of a finite number of mutually orthogonal primitive idempotent elements of $C$. Hence by Lemma 4.2 we see that $\Phi^{-1}(f) X$ and $\Phi^{-1}\left(f^{\prime}\right) X$ are finitely generated right $A$-modules.

From the assumption that $X$ is decomposed into a direct sum of finitely generated $A$-submodules $X_{\lambda}(\lambda \in \Lambda)$, it follows that there exist a finite number of elements $\lambda_{j}(j=1, \cdots, s)$ of $\Lambda$ such that $e X, e^{\prime} X, \Phi^{-1}(f) X$ and $\Phi^{-1}\left(f^{\prime}\right) X$ are all contained in $\sum_{j=1}^{s} \oplus X_{\lambda_{j}}$. Let $h$ be an element of $C$ which is a projection of $X$ onto $\sum_{j=1}^{s} \oplus X_{\lambda_{j}}$. Then by Lemma $4.3 h$ is an idempotent element which is a sum of a finite number of mutually orthogonal primitive idempotent elements of $C$. Hence by Lemma $4.2 \Phi(h) Y$ is a finitely generated right $B$-module.

The direct summands $e X$ and $e^{\prime} X$ of $X$ are both contained in $h X$, and hence they are also direct summands of $h X$. Hence $h X$ is a finitely generated, fully faithful right $A$-module.

On the other hand, we have $\Phi^{-1}(f)=h \Phi^{-1}(f), \Phi^{-1}\left(f^{\prime}\right)=h \Phi^{-1}\left(f^{\prime}\right)$ since $\Phi^{-1}(f) X$ and $\Phi^{-1}\left(f^{\prime}\right)$ are contained in $h X$. Hence $f=\Phi(h) f, f^{\prime}=\Phi(h) f^{\prime}$. Therefore $f Y$ and $f^{\prime} Y$ are contained in $\Phi(h) Y$, and, since they are direct summands of $Y$, they are also direct summands of $\Phi(h) Y$. This shows that $\Phi(h) Y$ is a finitely generated, fully faithful right $B$-module.

Thus we have arrived at the following situation: $h X$ is a finitely generated, fully faithful right $A$-module, $\Phi(h) Y$ is a finitely generated, fully faithful right $B$-module, $\Phi$ induces a ring-isomorphism of $h C h$ onto $\Phi(h) D \Phi(h)$, and $h C h$ $=\operatorname{End}_{A}(h X), \Phi(h) D \Phi(h)=\operatorname{End}_{B}(\Phi(h) Y)$.

We now apply Lemma 5.1 to the present situation. Then there exists a semi-linear isomorphism $\omega_{0}$ of $h X$ onto $\Phi(h) Y$ with a ring-isomorphism $\theta_{0}$ of $A$ onto $B$ such that

$$
\begin{array}{ll}
\omega_{0}(c x)=\Phi(c) \omega_{0}(x), & \text { for } x \in h X, c \in h C h, \\
\omega_{0}(x a)=\omega_{0}(x) \theta_{0}(a), & \text { for } x \in h X, a \in A .
\end{array}
$$

Since $e X$ is $A$-isomorphic to the right $A$-module $A$, there exists an element $x_{0}$ of $e X$ such that $e X=x_{0} A$ and $x_{0} a=0$ for $a \in A$ if and only if $a=0$. If we put $y_{0}=\omega_{0}\left(x_{0}\right)$, then we have

$$
\begin{aligned}
& \omega_{0}(e X)=\omega_{0}(h e h X)=\Phi(h) \Phi(e) \Phi(h) Y=\Phi(e) Y, \\
& \omega_{0}(e X)=\omega_{0}\left(x_{0} A\right)=\omega_{0}\left(x_{0}\right) \theta_{0}(A)=y_{0} B,
\end{aligned}
$$

and $y_{0} b=0$ for $b \in B$ if and only if $b=0$. Thus we have proved that $\Phi(e) Y$ is $B$-isomorphic to the right $B$-module $B$. Therefore by Lemma $4.1 Y$ is $D$-isomorphic to $D \Phi(e)$ as left $D$-modules. On the other hand, again by Lemma $4.1 X$ is $C$-isomorphic to $C e$ as left $C$-modules.

Of course, $\Phi$ induces a semi-linear isomorphism of the left $C$-module $C e$ onto the left $D$-module $D \Phi(e)$ by the correspondence $c \rightarrow \Phi(c)$ for $c \in C e$.

Therefore there exists a semi-linear isomorphism $\omega$ of the left $C$-module $X$ onto the left $D$-module $Y$ with the ring-isomorphism $\Phi$ of $C$ onto $D$ : 


$$
\omega(c x)=\Phi(c) \omega(x), \quad \text { for } x \in X, c \in C .
$$

For an element $a$ of $A$, the mapping $y \rightarrow \omega\left(\omega^{-1}(y) a\right)$ defines a $D$-endomorphism of $Y$ and hence by Lemma 4.1 there exists an element $\theta(a)$ of $B$ such that $\omega\left(\omega^{-1}(y) a\right)=y \theta(a)$. It is easy to see that $\theta$ is a ring-isomorphism of $A$ onto $B$. Thus we have

$$
\omega(x a)=\omega(x) \theta(a) \quad \text { for } x \in X, a \in A,
$$

and $\omega$ is a semi-linear isomorphism of the right $A$-module $X$ onto the right $B$-module $Y$ with a ring-isomorphism $\theta$ of $A$ onto $B$. This completes our proof of Theorem 3.3.

REMARK. In Theorems 3.2 and 3.3 the adjective "fully faithful" cannot be replaced by "faithful" in the usual sense.

Let $C$ be the subalgebra of the full matrix ring $(K)_{10}$ over a commutative field $K$ such that the elements

$$
\begin{aligned}
& e_{1}=c_{11}, \quad e_{2}=c_{22}+c_{55}, \quad e_{3}=c_{33}+c_{88}, \\
& e_{4}=c_{44}+c_{66}+c_{99}, \quad e_{5}=c_{77}, \quad e_{6}=c_{10,10}, \\
& c_{21}, \quad c_{31}, \quad c_{41}, \quad c_{42}+c_{65}, \quad c_{43}+c_{98}, \\
& c_{75}, \quad c_{76}, \quad c_{10,8}, \quad c_{10,9},
\end{aligned}
$$

form a $K$-basis of $C$, where $c_{i k}$ mean matrix units. The algebra is self-basic, and QF-3 and QF-1 in the sense of Thrall (cf. Morita [9, Example 2.2]). Let us put

$$
\begin{aligned}
X & =C e, & A & =e C e, & e & =e_{1}+e_{2}+e_{3} \\
Y^{\prime} & =C e^{\prime}, & B^{\prime} & =e^{\prime} C e^{\prime}, & e^{\prime} & =e+e_{5} \\
Y^{\prime \prime} & =C e^{\prime \prime}, & B^{\prime \prime} & =e^{\prime \prime} C e^{\prime \prime}, & e^{\prime \prime} & =e+e_{6} .
\end{aligned}
$$

Then $X$ is a fully faithful right $A$-module, and $Y^{\prime}$ (resp. $Y^{\prime \prime}$ ) is a faithful right $B^{\prime}$-module (resp. $B^{\prime \prime}$-module) which has a direct summand $B^{\prime}$-isomorphic to $B^{\prime}$ (resp. $B^{\prime \prime}$-isomorphic to $B^{\prime \prime}$ ). Since $C$ is $Q F-1$, we have $\operatorname{End}_{A}(X) \cong C$, $\operatorname{End}_{B^{\prime}}\left(Y^{\prime}\right) \cong C$, and $\operatorname{End}_{B^{\prime \prime}}\left(Y^{\prime \prime}\right) \cong C$. Hence $\operatorname{End}_{A}(X) \cong \operatorname{End}_{B^{\prime}}\left(Y^{\prime}\right)$ but there exists no semi-linear isomorphism of $X$ on to $Y$ since $A$ and $B^{\prime}$ are not ringisomorphic. On the other hand, if we correspond the $B^{\prime \prime}$-endomorphism of $Y^{\prime \prime}: y^{\prime \prime} \rightarrow c y^{\prime \prime}\left(y^{\prime \prime} \in Y^{\prime \prime}\right)$ to the $B^{\prime}$-endomorphism of $Y^{\prime}: y^{\prime} \rightarrow c y^{\prime}\left(y^{\prime} \in Y^{\prime}\right)$, we have a ring-isomorphism $\Phi$ of $\operatorname{End}_{B^{\prime}}\left(Y^{\prime}\right)$ onto $\operatorname{End}_{B^{\prime \prime}}\left(Y^{\prime \prime}\right)$ but there exists no semi-linear isomorphism $\omega$ of $Y^{\prime}$ onto $Y^{\prime \prime}$ such that $\Phi(f)=\omega \circ f \circ \omega^{-1}$ for each $f \in \operatorname{End}_{B^{\prime}}\left(Y^{\prime}\right)$ since such an $\omega$ would give a $C$-isomorphism of $Y^{\prime}$ onto $Y^{\prime \prime}$. (However, there is a semi-linear isomorphism of $Y^{\prime}$ onto $Y^{\prime \prime}$ which is induced by a ring-automorphism of $C$.)

6. A characterization of quasi-Frobenius rings. In this section we shall show under some condition that quasi-Frobenius rings are the most general type of rings for which the conclusion described in Theorem 1.2 holds. 
We shall say that $A$ has a duality for modules if there exists a duality between the category $\mathfrak{A M}^{*}$ of all finitely generated left $A$-modules and the category $\mathfrak{M}_{\Lambda}^{*}$ of all finitely generated right $A$-modules $\left({ }^{(}\right)$.

It is well known that an algebra of finite rank over a commutative field or a commutative ring with minimum condition has a duality for modules. It is an open question whether any ring with minimum condition has a duality for modules.

We shall prove

TheOREM 6.1. Suppose that for any finitely generated faithful right $A$-module $X$ and any finitely generated faithful right $B$-module $Y$ the existence of $a$ ringisomorphism of $\operatorname{End}_{A}(X)$ onto $\operatorname{End}_{B}(Y)$ implies the existence of a categoryisomorphism $T$ from $\mathfrak{M}_{A}$ onto $\mathfrak{M}_{B}$ such that $T(X)$ is $B$-isomorphic to $Y$. If there exist some $X$ and $Y$ such that $\operatorname{End}_{A}(X) \cong \operatorname{End}_{B}(Y)$, and if $A$ has a duality for modules, then $A$ and $B$ are quasi-Frobenius.

Proof. We shall use the notations described at the beginning of $\S 2$. Thus $A^{0}=e A e$ and $B^{0}=e^{\prime} B e^{\prime}$ are self-basic rings of $A$ and $B$ respectively, and $e$ and $e^{\prime}$ are idempotent elements of $A$ and $B$ respectively. Let $D=\left(D_{1}, D_{2}\right)$ be a duality between ${ }_{\Lambda} \mathfrak{M}^{*}$ and $\mathfrak{M}_{\Delta}^{*}$. We put $X_{0}=D_{1}(A e), Y_{0}=e^{\prime} B$, where $A e$ is considered as a left $A$-module and $Y$ is a right $B$-module. Then $\operatorname{End}_{A}\left(X_{0}\right)$ is inverse-isomorphic to the $A$-endomorphism ring of the left $A$-module $A e$ and the latter is inverse-isomorphic to $A^{0}$. Hence $\operatorname{End}_{A}\left(X_{0}\right) \cong A^{0}$. On the other hand, we have $\operatorname{End}_{B}\left(Y_{0}\right) \cong B^{0}$. Since there are some $X$ and $Y$ such that $\operatorname{End}_{A}(X) \cong \operatorname{End}_{B}(Y), A$ and $B$ are similar by Morita [8, Theorem 7.5], and hence $A^{0}$ is ring-isomorphic to $B^{0}$. Therefore we have $\operatorname{End}_{A}\left(X_{0}\right) \cong \operatorname{End}_{B}\left(Y_{0}\right)$. Hence from the assumption of the theorem it follows that there is a categoryisomorphism $T$ from $\mathfrak{M}_{A}$ onto $\mathfrak{M}_{B}$ such that $T\left(X_{0}\right)$ is $B$-isomorphic to $Y_{0}$. Since the left $A$-module $A e$ is projective, $X_{0}$ is injective and hence $T\left(X_{0}\right)$ is also injective, and consequently $Y_{0}$ is injective. Therefore by a theorem of Ikeda (for example, cf. Morita [8, Theorem 14.1]) $B$ is quasi-Frobenius, and $A$ is also quasi-Frobenius. This completes our proof.

From Theorems 6.1 and 1.2 we obtain a characterization of quasi-Frobenius rings.

Theorem 6.2. $A$ is a quasi-Frobenius ring if and only if $A$ has a duality for modules and for any finitely generated faithful right $A$-modules $X$ and $Y$ the existence of a ring-isomorphism of $\operatorname{End}_{A}(X)$ onto $\operatorname{End}_{A}(Y)$ implies the existence of a category-isomorphism $T$ from $\mathfrak{M}_{A}$ onto itself such that $T(X)$ is $A$-isomorphic to $Y$.

( ${ }^{\circ}$ A duality between $\mathbb{A}^{*}$ and $\mathfrak{M}_{4}$ is defined to be a pair $D=\left(D_{1}, D_{2}\right)$ of contravariant functors $D_{1}: \mathbb{M}^{*} \rightarrow \mathfrak{M}_{\Lambda}^{*}$ and $D_{2}: \mathfrak{M}_{\Lambda}^{*} \rightarrow \mathbb{M}^{*}$ such that the composite functors $D_{2} D_{1}$ and $D_{1} D_{2}$ are naturally equivalent to the identity functor. Cf. Morita [8]. 
7. The case of nonfaithful modules. In this section we shall prove the following theorems.

Theorem 7.1. Let $A$ be a uni-serial ring whose basic ring $A^{0}$ is decomposed into a direct sum of indecomposable two-sided ideals $A_{j}^{0}(j=1, \cdots, s)$ such that if $A_{i}^{0} / N_{i}^{0}$ is ring-isomorphic to $A_{j}^{0} / N_{j}^{0}$ then $A_{i}^{0}$ is ring-isomorphic to $A_{j}^{0}$ where $N_{\mathbf{k}}^{0}$ is the radical of $A_{\mathbf{k}}^{0}$. Let $B$ be similar to $A$. Then for a right $A$-module $X$ and $a$ right $B$-module $Y$ the existence of a ring-isomorphism of $\operatorname{End}_{A}(X)$ onto $\operatorname{End}_{B}(Y)$ implies the existence of a category-isomorphism $T$ from $\mathfrak{M}_{A}$ onto $\mathfrak{M}_{B}$ such that $T(X)$ is $B$-isomorphic to $Y$.

Theorem 7.2. Suppose that for any finitely generated right $A$-module $X$ and any finitely generated right $B$-module $Y$ the existence of a ring-isomorphism of $\operatorname{End}_{A}(X)$ onto $\operatorname{End}_{B}(Y)$ implies the existence of a category-isomorphism $T$ from $\mathfrak{M}_{A}$ onto $\mathfrak{M}_{B}$ such that $T(X)$ is $B$-isomorphic to $Y$. If there exist some $X$ and $Y$ such that $\operatorname{End}_{A}(X) \cong \operatorname{End}_{B}(Y)$ and if every residue class ring of $A$ has a duality for modules, then $A$ is a uni-serial ring with the structure described in Theorem 7.1 and $B$ is similar to $A$.

THEOREM 7.3. $A$ is a uni-serial ring with the structure described in Theorem 7.1 if and only if every residue class ring of $A$ has a duality for modules and for any finitely generated right $A$-modules $X$ and $Y$ the existence of a ring-isomorphism of $\operatorname{End}_{A}(X)$ onto $\operatorname{End}_{A}(Y)$ implies the existence of a category-isomorphism $T$ from $\mathfrak{M}_{A}$ onto itself such that $T(X)$ is $A$-isomorphic to $Y$.

Of course, Theorem 7.3 is a direct consequence of Theorems 7.1 and 7.2.

Proof of Theorem 7.2. We have only to consider the case where $A$ and $B$ are self-basic. In this case there is a ring-isomorphism $\theta$ of $A$ onto $B$, since $A$ is similar to $B$ as is seen from the existence of a category-isomorphism of $\mathfrak{M}_{A}$ onto $\mathfrak{M}_{B}$ by $[8$, Theorem 7.5$]$.

Let $I$ be any two-sided ideal of $A$. Then $A / I$ has a duality for modules and is self-basic. Since any finitely generated right $A / I$-module $X$ is a finitely generated right $A$-module and $\operatorname{End}_{A}(X) \cong \operatorname{End}_{A / I}(X)$, it follows from Theprem 6.1 that $A / I$ is quasi-Frobenius. Hence by a theorem of Ikeda [6] $A$ is uni-serial.

Now let $A=A_{1} \oplus \cdots \oplus A_{\mathrm{s}}$ be a decomposition of $A$ into a direct sum of indecomposable two-sided ideals of $A$. Let $N_{i}$ be the radical of $A_{i}$. Suppose that $A_{i} / N_{i}$ is ring-isomorphic to $A_{j} / N_{j}$.

We put $X=A_{i} / N_{i}, \quad Y=B_{j} / M_{j}$, where $B_{j}=\theta\left(A_{j}\right), \quad M_{j}=\theta\left(N_{j}\right)$. Then $\operatorname{End}_{A}(X)$ and $\operatorname{End}_{B}(Y)$ are ring-isomorphic. Since $A$ and $B$ are self-basic, there exists a semi-linear isomorphism $\omega$ of $X$ onto $Y$ with a ring-isomorphism $\phi$ of $A$ onto $B$. The correspondence $x \rightarrow \phi(x)(x \in A)$ induces a semi-linear isomorphism $\omega^{\prime}$ of $X$ onto the right $B$-module $\phi\left(A_{i}\right) / \phi\left(N_{i}\right)$ with a ring-isomorphism $\phi$ of $A$ onto $B$, and hence the right $B$-modules $Y$ and $\phi\left(A_{i}\right) / \phi\left(N_{i}\right)$ are $B$-isomorphic. Since a decomposition of $B$ into a direct sum of indecomposable 
two-sided ideals is unique, we see that $\phi\left(A_{i}\right)=B_{j}, \phi\left(N_{i}\right)=M_{j}$. Since $B_{j}=\theta\left(A_{j}\right)$, it follows that $A_{i}$ are ring-isomorphic to $A_{j}$. This proves Theorem 7.2.

Before proving Theorem 7.1 we shall give a lemma which was proved by Asano [1] for the special case where $X$ and $Y$ are finitely generated.

Lemma 7.4. Let $A$ be a primary uni-serial ring. Let $X$ and $Y$ be any two right $A$-modules. If $\operatorname{End}_{\Delta}(X)$ is ring-isomorphic to $\operatorname{End}_{A}(Y)$, then $X$ and $Y$ are $A$ isomorphic.

Proof. It is sufficient to prove the lemma for the case where $A$ is selfbasic, that is, $A$ is a completely primary uni-serial ring. If we put $I=\{a \mid x a=0$ for all $x \in X\}, J=\{a \mid y a=0$ for all $y \in Y\}$, then $X$ is a faithful right $A / I$-module and $Y$ a faithful right $A / J$-module. Since $A / I$ and $A / J$ are quasi-Frobenius and self-basic, we see by Theorem 1.3 that there is a semi-linear isomorphism $\omega$ of $X$ onto $Y$ with a ring-isomorphism $\theta$ of $A / I$ onto $A / J$. Since the totality of all two-sided ideals of $A$ is $\left\{N^{i} \mid i=0,1,2, \cdots\right\}$ where $N$ is the radical of $A$, we have $I=J$.

Let $X=\sum \oplus X_{\lambda}$ be a decomposition of $X$ into a direct sum of indecomposable right $A / I$-submodules. For each $\lambda$ there exists some $i$ such that $X_{\lambda} \cong A / N^{i}$, $I \subseteq N^{i}$. Then we have $\omega\left(X_{\lambda}\right) \cong A / N^{i}$ since $\theta\left(N^{i} / I\right)=N^{i} / I$. Thus $X_{\lambda} \cong \omega\left(X_{\lambda}\right)$ for each $\lambda$. Therefore $X$ and $Y$ are $A / I$-isomorphic, and consequently they are $A$-isomorphic. This proves Lemma 7.4.

Proof of Theorem 7.1. It is sufficient to prove the theorem for the case that $A$ and $B$ are self-basic. In this case there is a ring-isomorphism $\theta$ of $A$ onto $B$ since $A$ and $B$ are similar. Corresponding to the direct-sum decomposition of $A$ into indecomposable two-sided ideals: $A=A_{1} \oplus \cdots \oplus A_{\text {, we }}$ have a decomposition of $B: B=B_{1} \oplus \cdots \oplus B$, where $B_{i}=\theta\left(A_{i}\right)$.

Let $X$ be a right $A$-module and $Y$ a right $B$-module. If we put $X_{i}=X A_{i}$, $Y_{i}=Y B_{i}$, then we have the following decompositions:

$$
\begin{gathered}
X=X_{1} \oplus \cdots \oplus X_{s}, \quad Y=Y_{1} \oplus \cdots \oplus Y_{s}, \\
\operatorname{End}_{A}(X)=\operatorname{End}_{A_{1}}\left(X_{1}\right) \oplus \cdots \oplus \operatorname{End}_{A_{s}}\left(X_{s}\right), \\
\operatorname{End}_{B}(Y)=\operatorname{End}_{B_{1}}\left(Y_{1}\right) \oplus \cdots \oplus \operatorname{End}_{B_{s}}\left(Y_{s}\right) .
\end{gathered}
$$

If we set $I_{k}=\operatorname{Ann}\left(X_{k} ; A_{k}\right)\left({ }^{10}\right)$ and $J_{k}=\operatorname{Ann}\left(Y_{k} ; B_{k}\right)$ then we have

$$
\operatorname{End}_{A_{k}}\left(X_{k}\right)=\operatorname{End}_{A_{k} / I_{k}}\left(X_{k}\right) \text { and } \operatorname{End}_{B_{k}}\left(Y_{k}\right)=\operatorname{End}_{B_{k} / J_{k}}\left(Y_{k}\right) \text {. }
$$

Here we may assume without loss of generality that $X_{k} \neq 0, Y_{k} \neq 0, k=1, \cdots, s$. Then the $\operatorname{End}_{A_{k}}\left(X_{k}\right)$-endomorphism ring of the left $\operatorname{End}_{A_{k}}\left(X_{k}\right)$-module $X_{k}$ is indecomposable. Since $X_{k}$ is faithful as a left $\operatorname{End}_{A_{k}}\left(X_{k}\right)$-module, the ring End $_{A_{k}}\left(X_{k}\right)$ is indecomposable as a two-sided ideal of $\operatorname{End}_{A_{k}}\left(X_{k}\right)$. Consequently $\operatorname{End}_{A_{k}}\left(X_{k}\right)$ is indecomposable as a two-sided ideal of End $\operatorname{A}_{A}(X)$. Likewise $\operatorname{End}_{B_{k}}\left(Y_{k}\right)$ is indecomposable as a two-sided ideal of $\operatorname{End}_{B}(Y)$.

(10) By $\operatorname{Ann}(X ; A)$ we shall mean the annihilator ideal of $A$ for a right $A$-module $X$. 
Let $\Phi$ be a ring-isomorphism of $\operatorname{End}_{A}(X)$ onto $\operatorname{End}_{B}(Y)$. Since a decomposition of $\operatorname{End}_{B}(Y)$ into a direct sum of indecomposable two-sided ideals is unique (cf. Jacobson $[7$, p. 42]), there is a permutation $\pi$ of $(1,2, \cdots, s)$ such that $\Phi\left(\operatorname{End}_{A_{i}}\left(X_{i}\right)\right)=\operatorname{End}_{B_{j}}\left(Y_{j}\right)$ for $j=\pi(i)$.

Let $j=\pi(i)$.

Then $X_{i}$ is a faithful right $A_{i} / I_{i}$-module, $Y_{j}$ a faithful right $B_{j} / J_{j}$-module, and $A_{i} / I_{i}, B_{j} / J_{j}$ are self-basic quasi-Frobenius. Hence by Theorem 1.3 there is a semi-linear isomorphism $\omega_{i}$ of the right $A_{i} / I_{i}$-module $X_{i}$ onto the right $B_{j} / J_{j}$-module $Y_{j}$ with a ring-isomorphism $\theta_{i}$ of $A_{i} / I_{i}$ onto $B_{j} / J_{j}$. Let $N_{i}$ be the radical of $A_{i}$. Then $\left.I_{i} \subseteq N_{i}{ }^{11}\right)$, and hence $\theta^{-1} \circ \theta_{i}$ induces a ring-isomorphism of $A_{i} / N_{i}$ onto $A_{j} / N_{j}$. Therefore by the assumption on the structure of $A$ it is seen that there is a ring-isomorphism $\phi_{i}$ of $A_{i}$ onto $A_{j}$. If we put $\psi_{i}=\theta \circ \phi_{i}$, then $\psi_{i}$ is a ring-isomorphism of $A_{i}$ onto $B_{j}$. If we put further $y * a=y \psi_{i}(a)$ for $y \in Y_{j}, a \in A_{i}$, then $Y_{j}$ is a right $A_{i}$-module and its endomorphism ring is isomorphic to $\operatorname{End}_{B_{j}}\left(Y_{j}\right)$. Hence the $A_{i}$-endomorphism rings of the right $A_{i}$-modules $X_{i}$ and $Y_{j}$ are isomorphic. By Lemma 7.4 we see that $X_{i}$ and $Y_{j}$ are $A_{i}$-isomorphic. Thus there exists a semi-linear isomorphism $\omega_{i}^{\prime}$ of the right $A_{i}$-module $X_{i}$ onto the right $B_{j}$-module $Y_{j}$ with a ring-isomorphism $\psi_{i}$ of $A_{i}$ onto $B_{j}$. If we set

$$
\begin{aligned}
\psi\left(\sum_{i=1}^{s} a_{i}\right) & =\sum_{i=1}^{s} \psi_{i}\left(a_{i}\right), & a_{i} \in A_{i}, \\
\omega^{\prime}\left(\sum_{i=1}^{s} x_{i}\right) & =\sum_{i=1}^{s} \omega_{i}^{\prime}\left(x_{i}\right), & x_{i} \in X_{i},
\end{aligned}
$$

then $\omega^{\prime}$ is a semi-linear isomorphism of the right $A$-module $X$ onto the right $B$-module $Y$. This completes the proof of Theorem 7.1 .

8. Modules over a uni-serial ring. Finally, we shall prove the following theorems which determine the general type of rings having the property described in Lemma 7.4.

Theorem 8.1. If $A$ is a uni-serial ring whose basic-ring $A^{0}$ is decomposed into a direct sum of indecomposable two-sided ideals $A_{1}^{0}, \cdots, A_{s}^{0}$ such that $A_{i}^{0} / N_{i}^{0}$ is not ring-isomorphic to $A_{j}^{0} / N_{j}^{0}$ for $i \neq j$ where $N_{k}^{0}$ denotes the radical of $A_{k}^{0}$, then for any two right $A$-modules $X$ and $Y$ the existence of a ring-isomorphism of $\operatorname{End}_{A}(X)$ onto $\operatorname{End}_{A}(Y)$ implies the existence of an $A$-isomorphism of $X$ onto $Y$.

THEOREM 8.2. The converse of Theorem 8.1 holds in case $A$ has a duality for modules.

Proof of Theorem 8.1. We have only to prove the case where $A$ is selfbasic. In this case, let $A=A_{1} \oplus \cdots \oplus A_{8}$ be a decomposition of $A$ into a direct sum of indecomposable two-sided ideals; each $A_{i}$ is a completely

(iI) In case $I_{i}=A_{i}$ we have $J_{j}=B_{i}$ and hence $X_{i}=0, Y_{i}=0$. 
primary uni-serial ring and $A_{i} / N_{i}$ is not ring-isomorphic to $A_{j} / N_{j}$ for $i \neq j$ where $N_{k}$ is the radical of $A_{k}$.

Let $X$ and $Y$ be right $A$-modules such that $\operatorname{End}_{A}(X)$ is ring-isomorphic to $\operatorname{End}_{A}(Y)$. If we set $X_{i}=X A_{i}, Y_{i}=Y A_{i}, i=1, \cdots, s$, then we have the following direct-sum decompositions:

$$
\begin{aligned}
X & =\sum_{i=1}^{\dot{1}} \oplus X_{i}, & Y & =\sum_{i=1}^{s} \oplus Y_{i}, \\
\operatorname{End}_{A}(X) & =\sum_{i=1}^{\dot{1}} \oplus \operatorname{End}_{A_{i}}\left(X_{i}\right), & \operatorname{End}_{A}(Y) & =\sum_{i=1}^{s} \oplus \operatorname{End}_{A_{i}}\left(Y_{i}\right) .
\end{aligned}
$$

Here we may assume without loss of generality that $X_{i} \neq 0, Y_{i} \neq 0$, $i=1, \cdots, s$. Similarly as in the proof of Theorem 7.1 we see that there is a permutation $\pi$ of $(1, \cdots, s)$ such that $\operatorname{End}_{\Delta_{i}}\left(X_{i}\right)=\operatorname{End}_{\Delta_{j}}\left(Y_{j}\right)$ for $j=\pi(i)$. If we denote by $I_{i}$ resp. $J_{j}$ the annihilators $\operatorname{Ann}\left(X_{i} ; A_{i}\right) \operatorname{resp.} \operatorname{Ann}\left(Y_{j} ; A_{j}\right)$, then $A_{i} / I_{i}$ is ring-isomorphic to $A_{j} / J_{j}$. Since we have $I_{i} \subseteq N_{i}, J_{j} \subseteq N_{j}\left({ }^{11}\right)$, $A_{i} / N_{i}$ is also ring-isomorphic to $A_{j} / N_{j}$. From the assumption on the structure of $A$ it follows that $i=j$. Thus $\pi$ is the identity. Hence by Lemma 7.4 $X_{i} \cong Y_{i}$ as right $A_{i}$-modules, and consequently $X$ and $Y$ are $A$-isomorphic.

Proof of Theorem 8.2. As in the proof of Theorem 7.2 we have only to prove the theorem for the case where $A$ is self-basic.

From Theorem 6.2 it follows readily that $A$ is quasi-Frobenius. Let $N$ be the radical of $A$. Then by Nakayama [11, Part I, Theorem 6] we have $N^{i}=r\left(r\left(N^{i}\right)\right)=l\left(l\left(N^{i}\right)\right), i=1,2, \cdots$ where $r(S)=\{a \mid s a=0$ for every $s \in S\}$, $l(S)=\{a \mid a s=0$ for every $s \in S\}$ for a subset $S$ of $A$. Let $X$ be any finitely generated left $A$-module such that $N^{i} X=0$. Then we have $Y N^{i}=0$ for $Y=\operatorname{Char}_{A} X\left(=\operatorname{Hom}_{A}(X, A)\right)$; because if $x \in X, \alpha \in Y, m \in N^{i}$ then $m \alpha(x)$ $=\alpha(m x)=\alpha(0)=0$ and hence $\alpha(x) \in r\left(N^{i}\right)$, and consequently $(\alpha m)(x)=\alpha(x) m$ $=0$ since $N^{i}=r\left(r\left(N^{i}\right)\right)$. Similarly for any finitely generated right $A$-module $Y$ with $Y N^{i}=0$ the left $A$-module $\operatorname{Char}_{A} Y$ is annihilated by $N^{i}$ from the lefthand side. Therefore by associating with each finitely generated left or right $A / N^{i}$-module $X$ the $A$-character module $\operatorname{Char}_{A} X$ we obtain a duality between the category of all finitely generated left $A / N^{i}$-modules and the category of all finitely generated right $A / N^{i}$-modules. Thus $A / N^{i}$ has a duality for modules.

Now any finitely generated right $A / N^{i}$-module $X$ is considered as a finitely generated right $A$-module, and $\operatorname{End}_{A}(X)=\operatorname{End}_{A / N^{i}}(X)$. Hence by Theorem 6.2 it is seen that $A / N^{i}$ is quasi-Frobenius. In particular, $A / N^{2}$ is quasi-Frobenius. Hence by Nakayama [12] $A$ is a generalized uni-serial ring. We shall prove furthermore that $A$ is uni-serial. For this purpose by Nakayama [12] we have only to show that $B=A / N^{2}$ is uni-serial.

As is noted above $B$ is self-basic, quasi-Frobenius and generalized uniserial. Furthermore for any finitely generated right $B$-modules $X$ and $Y$ the 
existence of a ring-isomorphism of $\operatorname{End}_{B}(X)$ onto $\operatorname{End}_{B}(Y)$ implies the existence of a $B$-isomorphism of $X$ onto $Y$. Let $e$ be a primitive idempotent element of $B$ and $M$ the radical of $B$. Then we have $\operatorname{End}_{B}(e B / e M) \cong e B e / e M e$. On the other hand, $e M$ is the semi-simple part of the injective right $B$-module $e B$, and hence by our previous paper $\left[10\right.$, Theorem 4.2] $\operatorname{End}_{B}(e M) \cong e B e / e M e$; because if $e b e$ is an element of $B$ such that ebee $M=0$ then $e b e e B$ is a simple right $B$-module and hence $e b e e B \subseteq e M$. Therefore $e B / e M$ is $B$-isomorphic to $e M$. Consequently $B$ is uni-serial. Thus $A$ is uni-serial.

Let $A=A_{1} \oplus \cdots \oplus A_{\mathrm{s}}$ be a direct-sum decomposition of $A$ into indecomposable two-sided ideals of $A$. Here it is assumed that $A$ is self-basic. Moreover, since $A$ is uni-serial as is shown above, every residue class ring of $A$ has a duality for modules. Hence by Theorem $7.3 A$ has the structure described in Theorem 7.1; that is, if $A_{i} / N_{i}$ is ring-isomorphic to $A_{j} / N_{j}$, then $A_{i}$ is ring-isomorphic to $A_{j}$ where $N_{k}$ is the radical of $A_{k}$. Suppose that $A_{i} / N_{i}$ is ring-isomorphic to $A_{j} / N_{j}$ for $i \neq j$. Let us set $X=A_{i} / N_{i}, Y=A_{j} / N_{j}$; then $X$ and $Y$ are finitely generated right $A$-modules and $\operatorname{End}_{A}(X)$ is ring-isomorphic to $\operatorname{End}_{A}(Y)$, but $X$ and $Y$ are not $A$-isomorphic. Hence $A_{i} / N_{i}$ is not ring-isomorphic to $A_{j} / N_{j}$ for $i \neq j$. Thus the theorem is completely proved.

\section{REFERENCES}

1. K. Asano, Über verallgemeinerte Abelsche Gruppen mit Operatorenring und ihre Anwendungen, Jap. J. Math. 15 (1939), 231-253.

2. G. Azumaya, Corrections and supplementaries to my paper concerning Krull-RemakSchmidt's theorem, Nagoya Math. J. 1 (1950), 117-124.

3. - A duality theory for injective modules, Amer. J. Math. 81 (1959), 249-278.

4. R. Baer, Automorphism rings of primary abelian operator groups, Ann. of Math. 44 (1943), 192-227.

5. H. Cartan and S. Eilenberg, Homological algebra, Princeton Univ. Press, Princeton, 1956.

6. S. Ikeda, Some generalizations of quasi-Frobenius rings, Osaka Math. J. 3 (1951), 227-238.

7. N. Jacobson, Structure of rings, Amer. Math. Soc. Colloq. Publ. Vol. 37, Amer. Math. Soc., Providence, R. I., 1956.

8. K. Morita, Duality for modules and its applications to the theory of rings with minimum condition, Sci. Rep. Tokyo Kyoiku Daigaku 6, No. 150 (1958), 83-142.

9. - On algebras for which every faithful representation is its own second commutator, Math. Z. 69 (1958), 429-434.

10. K. Morita, Y. Kawada and H. Tachikawa, On injective modules, Math. Z. 68 (1957), 217-226.

11. T. Nakayama, On Frobeniusean algebras. I, Ann. of Math. 40 (1939), 611-633; II, Ann. of Math. 42 (1941), 1-21.

12. - Note on uni-serial and generalized uni-serial rings, Proc. Imp. Acad. Tokyo 16 (1940), 285-289.

13. M. Osima, Notes on basic rings. II, Math. J. Okayama Univ. 3 (1954), 109-119.

14. K. Shoda, Über die mit einer Matrix vertauschbaren Matrizen, Math. Z. 29 (1929), 696-712.

Tokyo University of Education,

TOKYO, JaPaN 